\title{
Daily versus post-dialysis administration of calcimimetics for the treatment of secondary hyperparathyroidism in haemodialysis patients: an interventional, multi-centre study
}

\author{
Tarek M Demerdash', Mostafa Abdelsalam², Mohammed Assem', Mahmoud M Shaheen', Mohamed A Marie
}

'Cairo University, Cairo, Egypt; ${ }^{2}$ Mansoura University, Mansoura, Egypt.

\begin{abstract}
Introduction: The treatment of secondary hyperparathyroidism (SHPT) and the mineral and bone disorder of chronic kidney disease (CKD-MBD) in patients on chronic haemodialysis (HD) may be hampered by poor adherence to medication. The aim of this study was to assess the effectiveness of supervised, post-HD administration of calcimimetics in controlling markers of CKD-MBD in these patie

Methods: This was a multi-centre prospective study that included adults treated at 16 private HD facilities in the Kingdom of Saudi Arabia with HD vintage $\geq 6$ months and proven SHPT. Patients with previous parathyroidectomy, baseline-corrected serum calcium < $8.4 \mathrm{mg} / \mathrm{dL}$ (2.1 mmol/L), tertiary HPT, and patients having other causes of hyper- or hypocalcaemia were excluded. As of I November 2017, study participants were shifted from daily dosing of calcimimetics to thrice weekly doses, at the end of each HD session. Intact parathyroid hormone (PTH), serum calcium, phosphorus and alkaline phosphatase levels, dosage of phosphate binders (calcium carbonate or sevelamer) and vitamin D analogues were-compared at baseline and 6 months after initiation of thrice weekly calcimimetic dosing.

Results: A total of 201 patients were included in the study. After 6 months, there was a significant reduction in calcimimetic dose $(P=0.003)$, associated with a significant decrease in PTH $(P=0.006)$ and alkaline phosphatase $(P=0.028)$. There were no significant changes in serum calcium or phosphorus, or calcium carbonate dose, but the sevelamer dose was significantly increased $(P=0.047)$.

Conclusions: Compared to daily dosing,thrice weekly calcimimetic dosing after HD was more effective in controlling markers of CKD-MBD. We recommend further evaluation in larger scale, randomized controlled studies.
\end{abstract}

Keywords: calciminetics, hyperparathyroidism; haemodialysis; Egypt.

\section{INTRODUCTION}

Secondary hyperparathyroidism (SHPT) and the other manifestations of the mineral and bone disorder of chronic kidney disease (CKD-MBD) are common and important complications in end-stage renal disease (ESRD) as it affects bone health and has a strong association with increased cardiovascular morbidity and mortality $[1,2]$. There are many options for treatment such as control of serum phosphate level by diet and phosphate binders to abolish the stimulatory effect of high serum phosphate on the parathyroid gland. Another approach is to suppress the stimulatory effect of hypocalcaemia on the parathyroid gland, either by correction of serum calcium levels using vitamin $D$ analogues or by stimulation of calcium sensing receptors on the parathyroid gland using drugs (calcimimetics), which in turn suppress parathyroid hormone (PTH) release.

While vitamin $\mathrm{D}$ and its analogues may be effective in managing SHPT, increases in serum calcium concentra- 
tions and a high calcium-phosphate product is a major limiting factor in their use [3]. With the addition of cinacalcet, a calcimimetic agent, this problem has been mitigated. Cinacalcet is approved by the US Food and Drug Administration and rapidly emerging as an important agent in treating SHPT and CKD-MBD in dialysis-dependent patients, though not in renal transplant patients or those with earlier stages of CKD [4]. Cinacalcet use leads to decreases in serum intact PTH levels $[5,6]$ and reduced risk of bone fractures [7]. Once-daily oral dosing is the standard prescription for cinacalcet $[8,9]$. The main adverse effects are gastrointestinal, mostly in the form of nausea and vomiting associated with high doses [10]. In haemodialysis (HD) patients who are usually using multiple medications, adherence to treatment is a challenge [II]. Because cinacalcet has a long half-life of more than 30 hours [12], it is possible to administer it on alternate days and so possibly improve patient adherence.

Our study aimed to assess the effectiveness of supervised, post-dialysis administration of calcimimetics in controlling markers of CKD-MBD in patients on maintenance HD.

\section{METHODS}

This was a multi-centre prospective study conducted at 16 private dialysis centres in the Kingdom of Saudi Arabia over a period of 6 months. Patients enrolled in the study all completed 6 months of follow-up. The inclusion criteria were adults on HD for at least 6 months, proven SHPT and willingness to provide informed consent. The patients in the study were receiving a daily dose of cinacalcet and were shifted to thrice weekly doses, starting I November 2017. We excluded patients with previous parathyroidectomy, baseline-corrected serum calcium $<8.4 \mathrm{mg} / \mathrm{dL}$ (2.1 mmold), tertiary HPT and those patients having other causes of hypercalcaemia or hypocalcaemia. Our patients were classified into three groups according to their baseline PTH levels: group I: PTH $\leq 600$ pg/mL; group II: PTH $\leq 600 \mathrm{pg} / \mathrm{mL}$ and $\leq 1200 \mathrm{pg} / \mathrm{mL}$; and group III: PTH $\leq 1200 \mathrm{pg} / \mathrm{mL}$.

Treatment protocol: The thrice weekly cinacalcet doses were administered by the nursing staff at the end of each haemodialysis session for the 6 months of the study. The dose was estimated from the prior daily dose and, in most cases, was double the daily dose. The dose was titrated every 4 weeks at 30-mg increments if the desired reduction in intact PTH was not achieved. The dose was not titrated further if (a) the maximum dose was reached, (b) corrected serum calcium decreased to below $8 \mathrm{mg} / \mathrm{dL}$ ( $2 \mathrm{mmol} / \mathrm{L})$, or (c) any adverse effects were noted. The thrice weekly cinacalcet dosages at the start and end of the study period were used for comparisons. The dose of vitamin D analogues and phosphate binders, whether calcium-based or not, could also be adjusted during the study.

Pre-dialysis PTH, serum calcium, phosphorus, alkaline phosphatase levels, and dosage of phosphate binders (calcium carbonate/sevelamer) and vitamin D analogue were compared at baseline and after 6 months.

Statistical analysis was performed using the Statistical Package for Social Sciences (version 20.0, SPSS Inc., Chicago, IL, USA). Quantitative variables were expressed as mean and standard deviation. Differences between continuous variables were examined with independent samples t- test for parameters with normal distribution and Mann-Whitney tests for non-normally distributed variables. Chi-square or Fisher's exact tests was used for comparison of quahitative variables, as appropriate. $\mathrm{P}$ values $<0.05$ were considered statistically significant.

Ethical approval: This work was approved by the King Fahd Medical City Institutional Review Board and was carried ut in accordance with the rules of the Helsinki Declaration (October 1983). Informed consent from the patients was obtained before the onset of the study.

\section{RESULTS}

A total of 201 patients were included; their mean age was $49.3 \pm 14.4$ years and $49.3 \%$ were male. The mean duration of daily cinacalcet treatment before switching to postdialysis therapy was 16.3 months (range 6-4I months).

Data on laboratory values and dosage of medications are summarized in Table I. There was no significant change in serum calcium or phosphate levels. There was a reduction in mean PTH levels from $1079 \mathrm{pg} / \mathrm{mL}$ at the start to $994 \mathrm{pg} / \mathrm{mL}$ after 6 months $(P=0.006)$, and a reduction in mean ALP levels from $190.2 \mathrm{U} / \mathrm{I}$ to $170.6 \mathrm{U} / \mathrm{I}(P=0.028)$. The mean doses of calcium carbonate and vitamin $D$ analogue (paricalcitol) were not different but the mean sevelamer dose increased from $4257 \mathrm{mg}$ to $4480 \mathrm{mg}$ daily $(P=0.047)$ and the thrice weekly cinacalcet dose decreased from $129.6 \mathrm{mg}$ to $87.2 \mathrm{mg}$ per dose $(P=0.003)$.

The number of patients who achieved the PTH target of $\leq 600 \mathrm{pg} / \mathrm{mL}$ increased from 36 at baseline to 63 at the end of the study (Table 2).

Post-dialysis dosing of cinacalcet was associated with reductions in $\mathrm{PTH}$ levels in all groups, defined by baseline PTH (Table 3). 
Table I. Effect of thrice weekly dosing of cinacalcet on laboratory values and dosage of medications.

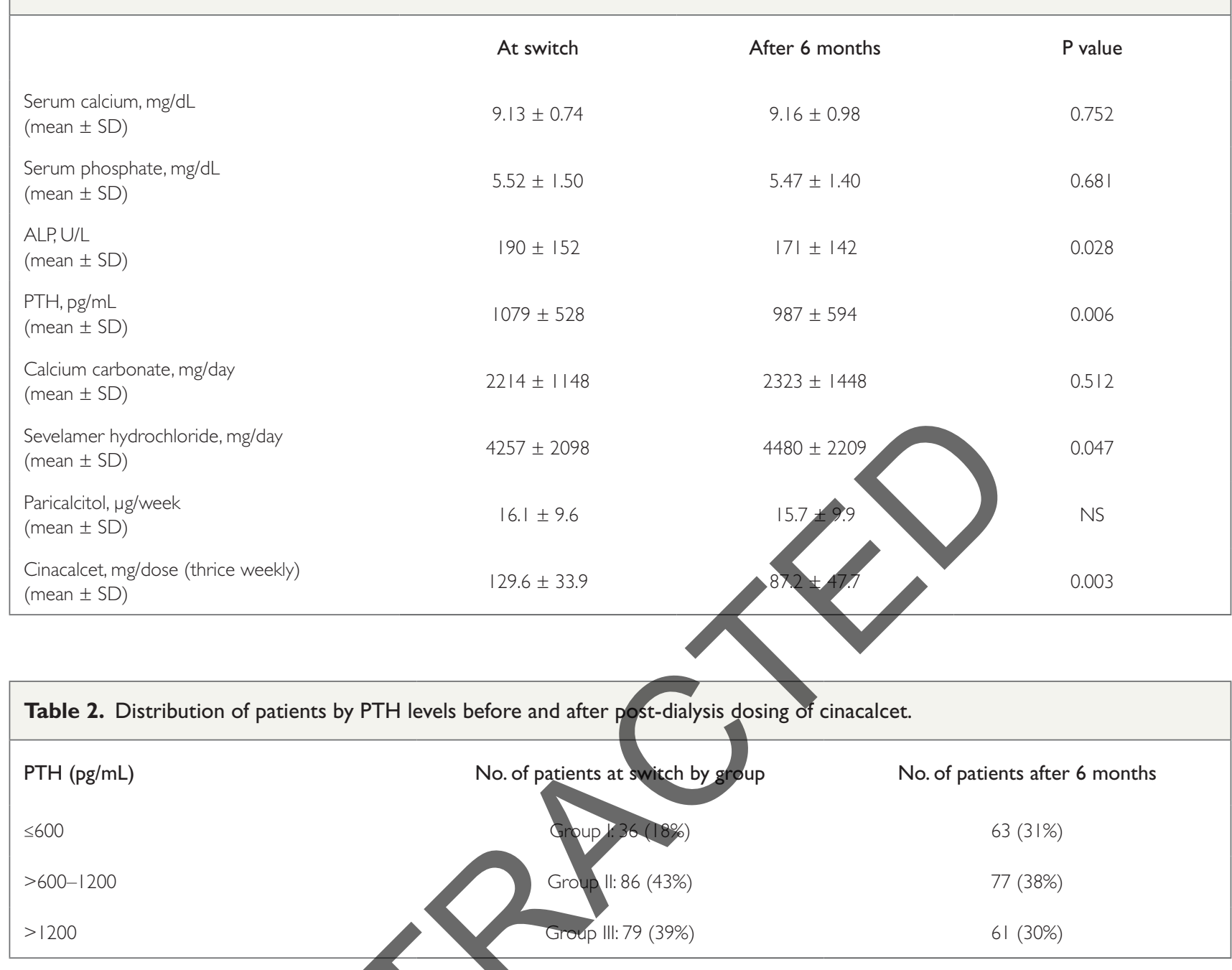

Table 3. Changes in PTH levels before and after post-dialysis dosing of cinacalcet in patient groups defined by baseline PTH.

\begin{tabular}{|c|c|c|c|c|}
\hline $\begin{array}{l}\text { Group } \\
\text { (no. of patients) }\end{array}$ & & $\begin{array}{l}\text { At switch }(\mathrm{pg} / \mathrm{mL}) \\
(\text { mean } \pm \mathrm{SD})\end{array}$ & $\begin{array}{c}\text { After } 6 \text { months }(\mathrm{pg} / \mathrm{mL}) \\
(\text { mean } \pm \mathrm{SD})\end{array}$ & $P$ value \\
\hline I (36) & $\leq 600$ & $391 \pm 160$ & $593 \pm 298$ & $<0.001$ \\
\hline II (86) & $>600-\leq 1200$ & $943 \pm 164$ & $808 \pm 446$ & 0.050 \\
\hline III (79) & $>1200$ & $1617 \pm 385$ & $1183 \pm 670$ & $<0.001$ \\
\hline
\end{tabular}

\section{DISCUSSION}

Some recent studies have assessed the effectiveness of intradialytic administration of calcimimetics and have reported good results, with satisfactory treatment adherence and without significant adverse effects [13]. Al Hilali et al. reported PTH suppression after administration of calcimimetics twice per week after dialysis, in a group of 27 haemodialysis patients [14]. In a small study of II patients, Haq et al. observed that post-dialysis administration of cinacalcet was as effective as daily home administration after 16 weeks of follow-up [15].
Our study included a larger number of patients and involved a longer period of follow-up. The idea of using thrice weekly post-dialysis cinacalcet is based on the pharmacokinetics and pharmacodynamics of the calcimimetic. The absolute bioavailability is $20-25 \%$, the half-life is $30-40$ hours, and steady-state concentrations in serum are achieved within 7 days. Following oral administration, cinacalcet suppresses PTH for almost 30 hours, with the maximum suppression achieved 2-6 hours after dosing. The pharmacokinetic profile in patients with renal insufficiency as well as those on dialysis, is comparable to that of healthy individuals [16]. 
We noted changes in the PTH and alkaline phosphatase values in our participants, with no significant changes in the values of serum calcium and phosphate. We also noted the increase in the required sevelamer doses, but no changes in the calcium carbonate or paricalcitol doses prescribed. Most importantly, the total dose of cinacalcet required per week was significantly reduced when compared to daily administration. This translates into substantial cost savings as cinacalcet is a very expensive drug.

Most randomized, controlled studies have documented that cinacalcet lowers serum calcium levels, improves hyperphosphataemia and reduces $\mathrm{PTH}$ [10]. Our findings were in line with this. However, regarding the increase in sevelamer dose, we are not aware of any reported data indicating an interaction between sevelamer and cinacalcet.

\section{CONCLUSIONS}

This study demonstrates that supervised calcimimetic dosing thrice weekly after HD was more effective than daily dosing in controlling markers of CKD-MBD. We have found that this approach also offers a cost saving option because of the reduction in total weekly dose. However, we recommend that large-scale trials be conducted to confirm these findings.

\section{Conflicts of interest}

The authors have no conflicts of

\section{REFERENCES}

I. Tentori F, Blayney MJ, Albert JM, Gillespie BW, Kerr PG, Bommer J, et al. Mortality risk for dialysis patients with different levels of serum calcium, phosphorus, and PTH: the Dialysis Outcomes and Practice Patterns Study (DQPRS). Am J Kidney Dis. 2008; 52(3):5 19-530.

2. Melamed ML, Eustace JA, Plantinga L, Jaar BG, Fink NE, Coresh J, et al. Changes in serum calcum, phosphate, and PTH and the risk of death in incident dialysis patients: a longitudinal study. Kidney Int. 2006; 70(2):351-357.

3. Sprague SM, Llach F, Amdahl M, Taccetta C, Batlle D. Paricalcitol versus calcitriol in the treatment of secondary hyperparathyroidism Kidney Int. 2003; 63(4): I 483- 1490.

4. Verheyen N, Pilz S, Eller K, Kienreich K, Fahrleitner-Pammer A, Pieske $B$, et al. Cinacalcet hydrochloride for the treatment of hyperparathyroidism. Expert Opin Pharmacother. 2013; 14:793-806.

5. Barman Balfour JA and Scott L J. Cinacalcet hydrochloride. Drugs. 2005; 65(2):27I-8I.

6. Urena P, Jacobson SH, Zitt E, Vervloet M, Malberti F, Ashman N, et al. Cinacalcet and achievement of the NKF/K-DOQI recommended target values for bone and mineral metabolism in real-world clinical practice - the ECHO observational study. Nephrol Dial Transplant. 2009; 24(9):2852-9.

7. Moe SM, Abdalla S, Chertow GM, Parfrey PS, Block GA, CorreaRotter R, et al. Effects of cinacalcet on fracture events in patients receiving haemodialysis: The EVOLVE trial. J Am Soc Nephrol.
8. Kidney Disease: Improving Global Outcomes CKD-MBD Work Group. KDIGO clinical practice guideline for the diagnosis, evaluation, prevention, and treatment of chronic kidney diseasemineral and bone disorder (CKD-MBD). Kidney Int Suppl. 2009; ( I I3):SI-130.

9. Harris RZ, Padhi D, Marbury TC, Noveck RJ, Salfi M, Sullivan JT. Pharmacokinetics, pharmacodynamics, and safety of cinacalcet hydrochloride in haemodialysis patients at doses up to $200 \mathrm{mg}$ once daily. Am J Kidney Dis. 2004; 44(6): 1 070-6.

10. Peacock M, Bolognese MA, Borofsky M, Scumpia S, Sterling LR, Cheng $S$, et al. Cinacalcet treatment of primary hyperparathyroidism: biochemical and bone densitometric outcomes in a five-year study. J Clin Endocrinol Metab. 2009; 94(I 2):4860-7.

II. Zwiech R, Chrul S. The influence of side effects related to cinacalcet therapy on compliance in haemodialysis patients suffering from secondary hyperparathyroidism. Wiad Lek. 20 I2; 65(3): I 45- I 50.

12. Padhi D, Harris R. Clinical pharmacokinetic and pharmacodynamic profile of cinacalcet hydrochloride. Glin Pharmacokinet. 2009; 48(5):303-31।.

13. Esteve Simo V, Moreno-Guzmán F, Martínez Calvo G, Fulquet Nicolas M, Pou Potau M, Macias-Toro), et al. Administration of calcimimetics after dialysis: Same effectiveness, better gastrointestinal tolerability. Nephrologia. 2015, 35(4):403-409.

14. Al Hilali N, Hussain N. Kay YA, Al Azmi M. A novel dose regimen of cinacalcet in the treatment of severe hyperparathyroidism in haemodialysis patients. Saudi J Kidney Dis Transpl. I; (22):448-455.

15. Haq N, Chaaban A, Gerban N, Khan I, Abbachi F, Hassan M, et al. A prospective randomized pilot study on intermittent post-dialysis dosing of cinacalcet. Int Urol Nephrol. 20 I 4; 350: 15 I 6- I 525.

16. Padhi D, Harris R. Clinical pharmacokinetics and pharmacodynamic profile of cinacalcet hydrochloride. Clin Pharmacokinet. 2009; 48:303-311. 